features. Electroencephalographic response to hyperventilation was exaggerated in eight subjects $(21 \%)$ but was not related to the occurrence of a headache. Photic stimulation showed high frequency driving in all 16 patients who developed headache but in only 14 out of 22 (64\%) who did not develop headache (Lai, $\mathrm{C}$ et al. Clinical and electrophysiological responses to dietary challenge in migraineurs. Headache March 1989; 29:180-186).

COMMENT: Foods are commonly cited by patients as the cause of same migraine attacks. Tyramine is present in high concentrations in certain substances frequently producing migraine (various cheeses, beer and wine). Electroencephalographic abnormalities are found during asymptomatic periods in patients with migraine, and focal and unilateral delta rhythms have been described in patients with migraine during symptomatic states. Paroxysmal epileptiform discharges are unusual in adults with migraine but not uncamon in children. Temporal relationships between headache and severe episodic EEG abnormality ("ictal headache") have been reported (Isler $\mathrm{H}$ et al in Andermann F, Lugaresi E (eds): Migraine and epilepsy, Boston, Butterworths, 1987).

ASPIRIN PROPHYLAXIS IN GHRONIC PAROXYSMAL HEMICRANIA

A nine year old child with chronic paroxysmal hemicrania (CPH) was treated successfully using small dose aspirin prophylaxis at the California Medical Clinic for Headache, Encino, and the Harbor-UCLA Medical Center, Torrance, CA. At tacks occurred every $1 \frac{1}{2}$ hours throughout the day and awakened him from a sleep at night. They lasted a minimum of ten minutes and a maximum of 20 minutes and were localized to the left retroorbital and supraorbital areas. Pain was excruciating and nonthrobbing and was associated with ipsilateral lacrimation, nasal stuffiness, ptosis, and conjunctival injection. No relief was obtained with acetaminophen or phenobarbital. Baby aspirin (243 $\mathrm{mg}$ b.i.d. prevented the headaches and the dosage was decreased to $162 \mathrm{mg}$ b.i.d. without further attacks. The aspirin was discontinued after three months without recurrence of headaches. The authors consider that this case is the first report of chronic paroxysmal hemicrania observed in a child, the earliest onset of $\mathrm{CPH}$, and the first case obtaining relief from low dose prophylactic aspirin therapy. The effective daily dose of aspirin used (14.7 $\mathrm{mg} / \mathrm{kg}$ ) was less than the lowest mean level at risk for Reye's syndrome (25.1 $\mathrm{mg} / \mathrm{kg}$ ). (Kudrow D.B., Kudrow L. Successful aspirin prophylaxis in a child with chronic paroxysmal hemicrania. Headache March 1989; 29:280-281).

COMENT: Indamethacin prophylaxis is considered the treatment of choice in adults with chronic paroxysmal hemicrania whereas salicylates are usually ineffective. Aspirin prophylaxis for chronic headache in children would not be a popular therapy generally because of the concern about Reye's syndrome.

\title{
MIGRAINE AS A NEUROVASCULAR METABOLIC DISORDER
}

Brain oxidative metabolism has been studied in nine patients with classical migraine at the Neurological Institute, University of Bologna, Italy. An increase in plasma lactate was found after standardized muscular effort and deficits of various mitochondrial respiratory chain enzymes in muscle biopsies occurred in 7 . Two patients had $72 \%$ and $60 \%$ depression of 
cytochrome C oxidase activity; 1 also showed ragged red fibers and subsarcolemmal clusters of giant mitochondria with paracrystalline inclusions. The findings indicated a dysfunction of mitochondrial energy metabolism and suggested that migraine is the result of a defect of brain oxidative metabolism. The authors conclude that neural energetic lability, especially if coupled with vascular metabolic dysfunction, could result - particularly under stressful conditions - in the neurological deficits of classical migraine. It was their hypothesis that a metabolic oxidative defect involving brain cells and possibly brain vessels represents the critical factor predisposing migraineurs to transient or persistent neurological deficits. (Montagna $\mathrm{P}$ et al. Migraine as a defect of brain oxidative metabolism: A hypothesis. J Neurol Feb 1989; 236:124-125).

COMMENT. The neurological deficits of migraine have been attributed to brain ischemia or a primary derangement of brain metabolism. The progression of the aura and the prodromal symptams are difficult to reconcile with a purely vascular problem and changes in platelet function suggest a diffuse extracerebral metabolic disturbance. Migraine attacks sametimes occur as complications of mitochondrial encephalamyopathies which reinforces the suggestion that migraine is the result of a defect of brain oxidative metabolism.

\section{DIET AND BEHAVIOR}

ELIMINATION DIETS IN PRE-SOHOOL-AGED HYPERACTIVE BOYS

The effect of an experimental elimination diet was examined in 24 hyperactive boys aged 3.5 to 6 years at the Alberta Children's Hospital, and the Learning Center, Calgary, Alberta, Canada. The diet was broader than those studied previously in that it eliminated not only artificial colors and flavors but also chocolate, monosodium glutamate, preservatives, caffeine, and any substance that the families reported might affect the child. It was low in simple sugar (mono- and disaccharaides) and dairy-free if an allergy to milk was suspected. A within-subject cross-over design was divided into 3 periods: a baseline of three weeks, a placebo-control period of three weeks, and an experimental diet period of four weeks. Approximately $42 \%$ (10) of the children showed 50\% improvement in behavior on the elimination diet; an additional $16 \%$ (4) had lesser degrees of improvement (12\%) with no placebo effect. Headache was less frequently reported during the diet period compared to placebo but not less than the base-line phase. Other nonbehavioral variables such as night awakenings and halitosis tended to improve during the dietary treatment phase. (Kaplan BJ et al. Dietary replacement in preschool-aged hyperactive boys. Pediatrics Jan 1989; 83:7-17).

COMMENT. These results of replacement diets indicate larger response rates than challenge studies with specific items. Further studies of additive-free and hypoallergenic-sugar-restricted diets are warranted in the management of attention deficit disorders with hyperactivity, and headache and sleep disorders, particularly in preschool children. 